This document is the accepted manuscript version of the following article: Moroz, P., Liyanage, G., Kholmicheva, N. N., Yakunin, S., Rija1, U., Uprety, P., ... Zamkov, M. (2014). Infrared emitting Pbs nanocrystal solids through matrix encapsulation. Chemistry of Materials, 26(14), 4256-4264. https://doi .org/10.1021/cm501739h 


\title{
Infrared Emitting PbS Nanocrystal Solids through
}

\section{Matrix Encapsulation.}

\author{
Pavel Moroz ${ }^{1,3 \dagger}$, Geethika Liyanage ${ }^{3 \dagger}$, Natalia N. Kholmicheva ${ }^{1,3}$, Sergii Yakunin $^{4,5}$, Upendra Rijal , \\ Prakash Uprety ${ }^{3}$, Ebin Bastola ${ }^{3}$, Bryan Mellott ${ }^{2}$, Kamal Subedi $^{3}$, Liangfeng Sun ${ }^{1,3}$, Maksym V. \\ Kovalenko ${ }^{4,5}$, Mikhail Zamkov ${ }^{1,3, *}$.
}

The Center for Photochemical Sciences ${ }^{1}$, Department of Chemistry ${ }^{2}$, and Department of Physics ${ }^{3}$, Bowling Green State University, Bowling Green, Ohio 43403. Department of Chemistry and Applied Biosciences $^{4}$, ETH Zürich, Zürich, 8093, Switzerland. EMPA-Swiss Federal Laboratories for Materials Science and Technology 5 , Dübendorf, 8600, Switzerland.

${ }^{\dagger}$ These authors contributed equally to this work.

*zamkovm@bgsu.edu

RECEIVED DATE (to be automatically inserted after your manuscript is accepted if required)

\begin{abstract}
Colloidal semiconductor nanocrystals (NCs) are emerging as promising infrared-emitting materials, which exhibit spectrally-tunable fluorescence, and offer the ease of thin film solution processing. Presently, an important challenge facing the development of nanocrystal infrared emitters concerns the fact that both the emission quantum yield and the stability of colloidal nanoparticles become compromised when nanoparticle solutions are processed into solids. Here, we address this issue by developing an assembly technique that encapsulates infrared-emitting $\mathrm{PbS}$ NCs into crystalline $\mathrm{CdS}$ matrices, designed to preserve $\mathrm{NC}$ emission characteristics upon film processing. An important feature of the reported approach is the heteroepitaxial passivation of nanocrystal surfaces with a CdS


semiconductor, which shields nanoparticles from the external environment leading to a superior thermal and chemical stability. Here, the morphology of these matrices was designed to suppress the nonradiative carrier decay, whereby increasing the exciton lifetime up to $1 \mu \mathrm{s}$, and boosting the emission quantum yield to an unprecedented 3.7\% for inorganically encapsulated $\mathrm{PbS} \mathrm{NC}$ solids.

\author{
KEYWORDS. Lead sulfide, colloidal quantum dots, inorganic matrix, time-resolved \\ fluorescence, quantum yield.
}

Near-infrared light emitting materials operating in the 700-2000 nm spectral range are commonly employed by a wide scope of applications ranging from biomedical imaging to telecommunication technologies. This spectral window is notoriously difficult to access since the emission quantum yield of most organic or inorganic infrared materials is substantially lower than that of visible-range counterparts. In light of this constraint, semiconductor nanocrystals exhibiting bright and tunable infrared fluorescence represents a promising material class for the development of near-IR technologies. ${ }^{1}$ Of a particular interest are lead chalcogenide nanoparticles, where the quantum confinement of photoinduced charges enables an emission quantum yield (QY) of up to $50 \%$ in solution, ${ }^{2-4}$ far above the typical QY range of indirect gap Ge or Si semiconductors.

A significant challenge for optical applications of colloidal NCs concerns their integration into transparent host matrices, which preserve their size-dependent optical properties. Besides enabling bright band gap emission, these matrices should be designed to maintain the monodispersity of embedded nanocrystals, exhibit a good thermal and chemical stability, and possess a high refractive index in order to be pertinent for utilization in waveguide-based applications. The existing matrix encapsulation strategies relying on organic polymer mixes ${ }^{5-10}$ are reasonably successful in converting 
nanoparticle solutions into device-compatible films, but suffer from poor thermal stability as well as polymer/semiconductor phase separation. Both of these issues can be traced back to the existence of molecular ligands preserved on surfaces of matrix-encapsulated nanocrystals. These molecules tend to desorb when thermally or electrically stimulated and are known to cause poor nanoparticle miscibility with the polymer environment. The employment of sol-gel titania matrices ${ }^{11-13}$ has helped resolving some of these difficulties through the use of titania functional groups tethered directly to alcoholterminated amine ligands, however, this and other emerging strategies ${ }^{14-16}$ still suffered from poor stability of ligand-terminated NC surfaces. One viable solution to the stability problem was recently offered by the work of Kovalenko et $a .^{2}{ }^{2}$ who have employed an encapsulation strategy utilizing metal chalcogenide complexes (MCC). ${ }^{17,18}$ This methodology relied on sintering of hybrid ligands comprising MCCs into $\mathrm{As}_{2} \mathrm{~S}_{3}$ amorphous matrices, which gave rise to an all-inorganic film architecture exhibiting compelling stability and a large refractive index.

Despite recent advances in enhancing the stability of nanocrystal solids, ${ }^{2,19-21}$ the emission characteristics of these metamaterials require further improvement. In the visible range, the fluorescence quantum yield (QY) of titania-encapsulated CdSe NCs was reported to undergo a seven-fold drop upon transferring of nanoparticles into a solid form. ${ }^{12}$ Similar trend was observed in the case of infrared emitting NCs. A spincoated film of PbSe NCs typically exhibits an emission QY of $1-2 \%{ }^{22}$ which constitutes a significant reduction from a $50 \%$ value observed for these nanoparticles in solution. ${ }^{23}$ The QY of PbS NC solids can be increased to $4-12 \%$ through the use of polymer matrices, ${ }^{9}$ however, a brief exposure of these films to $\mathrm{T}=100 \mathrm{C}^{\circ}$ or above results in a rapid loss of emission. Inorganic films of infrared nanocrystals are more stable but less emissive. The fluorescence QY of $\mathrm{As}_{2} \mathrm{~S}_{3}$-encapsulated PbS NC solids, is around $0.1 \% .{ }^{24}$ The addition of a CdS shell prior to capping with MCC ligands results in an 
improved QY value, however, sintering of colloidal nanocrystals into a glassy matrix ${ }^{1}$ is still accompanied by the 4.5-time drop in the exciton lifetime.

Amongst the primary non-radiative processes that compete with the radiative decay of matrixencapsulated NCs, two mechanisms of exciton dissociation that have been previously identified to contribute the most into quenching of the nanoparticle fluorescence. ${ }^{25,26}$ First, dot-to-dot charge transfer can lead to the formation of a "dark" excited state, whereby reducing the probability of the radiative recombination. Likewise, an interparticle energy transfer increases the probability of defect filling due to multiple dot-to-dot transitions, thus contributing to a non-radiative decay. Second, charge trapping on dangling bonds and nanocrystal/matrix interfaces can cause exciton dissociation followed by a nonradiative relaxation of carriers. With respect to the interparticle charge transfer process, the rate of dotto-dot carrier tunneling was previously shown to have a nearly exponential dependence on the width and the height $(\sqrt{\Delta E})$ of the potential barrier separating adjacent nanocrystals. ${ }^{27}$ Therefore, the charge transfer pathway of exciton dissociation can be readily suppressed by choosing a wide gap matrix material or by employing a sufficient interparticle separation.

Trapping of excited carriers on surfaces and interfaces of encapsulated nanoparticles, however, represents a more serious problem. In the case of polymer or titania matrices, the surfaces of encapsulated nanocrystals retain original, long-chain organic ligands to help lower the density of surface traps. ${ }^{12}$ This strategy, however, suffers from the propensity of ligand molecules to detach from NCs as a result of a thermal or electrical stimulation. The desorption of ligands inevitably compromises the stability of the NC emission in a film, regardless of the stability of the matrix medium. In the case of glassy matrices, hybrid or fully inorganic ligands serve as a surface passivation layer, ${ }^{1,28-33}$ which warrants a compelling thermal stability. The glass sintering step, however, may result in an imperfect surface passivation, ${ }^{34}$ which could be partly responsible for a low emission yield observed in the case of $\mathrm{As}_{2} \mathrm{~S}_{3}$-encapsulated $\mathrm{PbS} \mathrm{NC}$ solids. Ideally, well defined stoichiometric relationships should exist 
between encapsulated nanoparticles and a matrix medium, which itself should exhibit a high thermal and chemical stability.

Here, we demonstrate a solution-phase assembly of $\mathrm{PbS}$ nanocrystals into inorganic solids exhibiting bright infrared emission and superior thermal stability. The reported methodology takes the advantage of crystalline semiconductor matrices, heteroepitaxially bonded to surfaces of encapsulated $\mathrm{PbS}$ nanocrystals. Such passivation strategy promotes a reduced density of surface traps, which is manifested in this work through the observation of a compelling emission quantum yield. The suppressed defect trapping is also apparent through the detection of long exciton lifetimes approaching 1 microsecond for best-performing films. This constitutes a noticeable improvement over NC solids assembled via conventional ligand-linking or amorphous-matrix strategies.

\section{Results and Discussion.}

The matrix encapsulation technique developed in this work builds upon a recently reported semiconductor matrix embedded nanocrystal array (SMENA) approach, ${ }^{35,44}$ which allows for a gap-free inclusion of colloidal NCs into host matrices of wide band gap semiconductors. Typically, nanocrystals are encapsulated using a three-step procedure (see Fig. 1a). First, hot-injection chemistry is used to grow monodisperse PbS NCs, onto which a shell of a lattice-matched semiconductor (CdS) is deposited. The matrix semiconductor is chosen to form a type I band edge alignment with $\mathrm{PbS}$, thus promoting core-localization of both carrier types (Fig. 2a). In the second step, core/shell $\mathrm{NCs}(\mathrm{PbS} / \mathrm{CdS})$ are spincoated onto a suitable substrate, capped with thermally degradable ligands, and subsequently heated to $140-160{ }^{\circ} \mathrm{C}$ to promote the ligand removal (as monitored by FTIR) and to induce merging of respective shells (CdS-CdS) via the crystallographic fusion process. In the final step, the pores of the $\mathrm{CdS}$ matrix are filled with an additional $\mathrm{CdS}$ material through the successive ionic layer adsorption and reaction (SILAR) method. ${ }^{36}$ This step helps passivating exposed surfaces of the CdS matrix (thus neutralizing carrier traps) and increases an average refractive index (n) of the film. The pore filling step also promotes an enhanced stability of the $\mathrm{PbS} \mathrm{NC}$ array. 

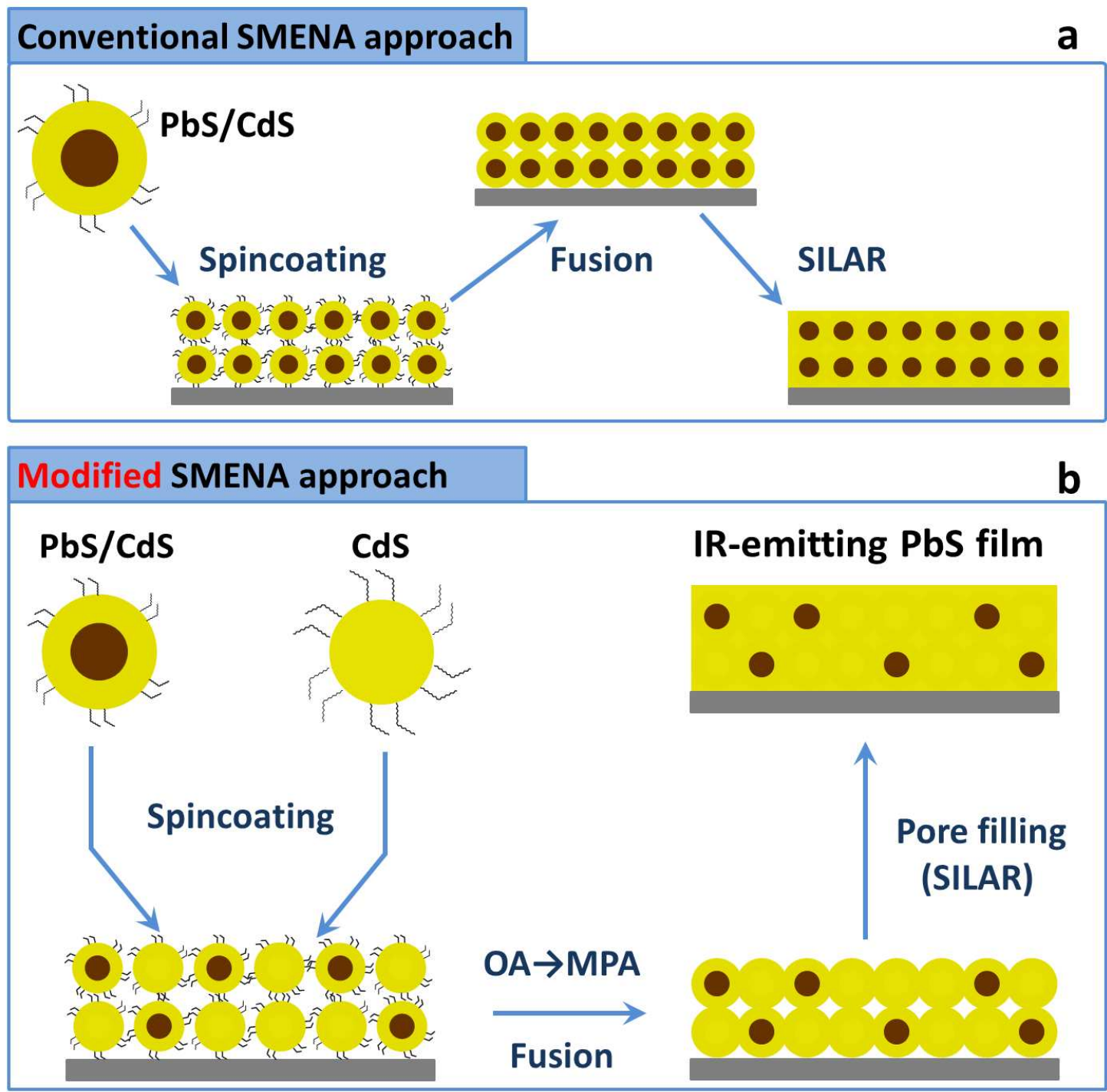

Figure 1. (a). Illustration of the key steps involved in the development of conventional Semiconductor Matrix-Encapsulated Nanocrystal Arrays (SMENA). These stages include colloidal synthesis of $\mathrm{PbS} / \mathrm{CdS}$ core/shell NCs (step 1), spincoating or dip-coating of NC films (step 2), exchange of bulky ligands with thermally degradable MPA molecules, crystallographic fusion of core/shell NCs performed using a layer-by-layer deposition (steps 3), and in-filling the pores of the resulting matrix 
with additional CdS (step 4). (b). In a modified SMENA approach, PbS/CdS NCs are mixed with CdSonly NCs (of a similar diameter) in the first step.

To enhance the radiative portion of the excited state decay in matrix-encapsulated nanocrystals, the localization of photoinduced charges should be increased. In a conventional SMENA approach, ${ }^{34}$ this goal is achieved by augmenting the thickness of the shell, $\Delta H$, in core/shell precursor NCs, which determines the ultimate value of the minimal edge-to-edge distance between $\mathrm{PbS}$ domains in the film $\left(\mathrm{R}_{\text {edge }}=2 \times \Delta H\right)$. Indeed, larger $\mathrm{R}_{\text {edge }}$ results in the suppression of the interparticle charge and energy transfer processes, leading to enhanced probability of the radiative recombination. This situation is illustrated in Fig. 2b, showing the relationship between the interparticle charge transfer rate and the $\mathrm{R}_{\text {edge }}$ distance in the film. It is assumed that the charge tunneling rate has a single exponential dependence on the width of the potential barrier separating neighboring nanocrystals. According to Wentzel-KramersBrillouin (WKB) approximation, this rate is proportional to $\Gamma \sim \exp \left(-2\left(2 m \Delta E / \hbar^{2}\right) R_{\text {edge }}\right)$, where $\Delta E$ is the height of the potential barrier between electron or hole states in adjacent NCs. The regime of strong charge localization is achieved when the coupling energy $\beta=\hbar \omega \Gamma$ drops below $k T$, known as Mott insulator regime. Since the carrier localization is more pronounced in dots with a larger core size (Fig. 2a), the characteristic $R_{\text {edge }}$ distance at which such transition occurs diminishes with the growing diameter of the $\mathrm{PbS}$ domain. This trend is clearly seen in Fig. $2 b$ through the comparison of the inter-dot coupling energy for the cases of 4-nm and 6-nm diameter PbS NCs embedded into CdS matrices. For example, the value of $\mathrm{R}_{\text {edge }}$ associated with the insulator transition in 4-nm-PbS/CdS solids is about 2 times greater than that of $6-\mathrm{nm}-\mathrm{PbS} / \mathrm{CdS}$. We note that while $\mathrm{x}$-axis in Fig. $2 \mathrm{~b}$ is given only in relative units of scale, it preserves the correct lengths ratio corresponding to insulator transitions between different dot diameters. 

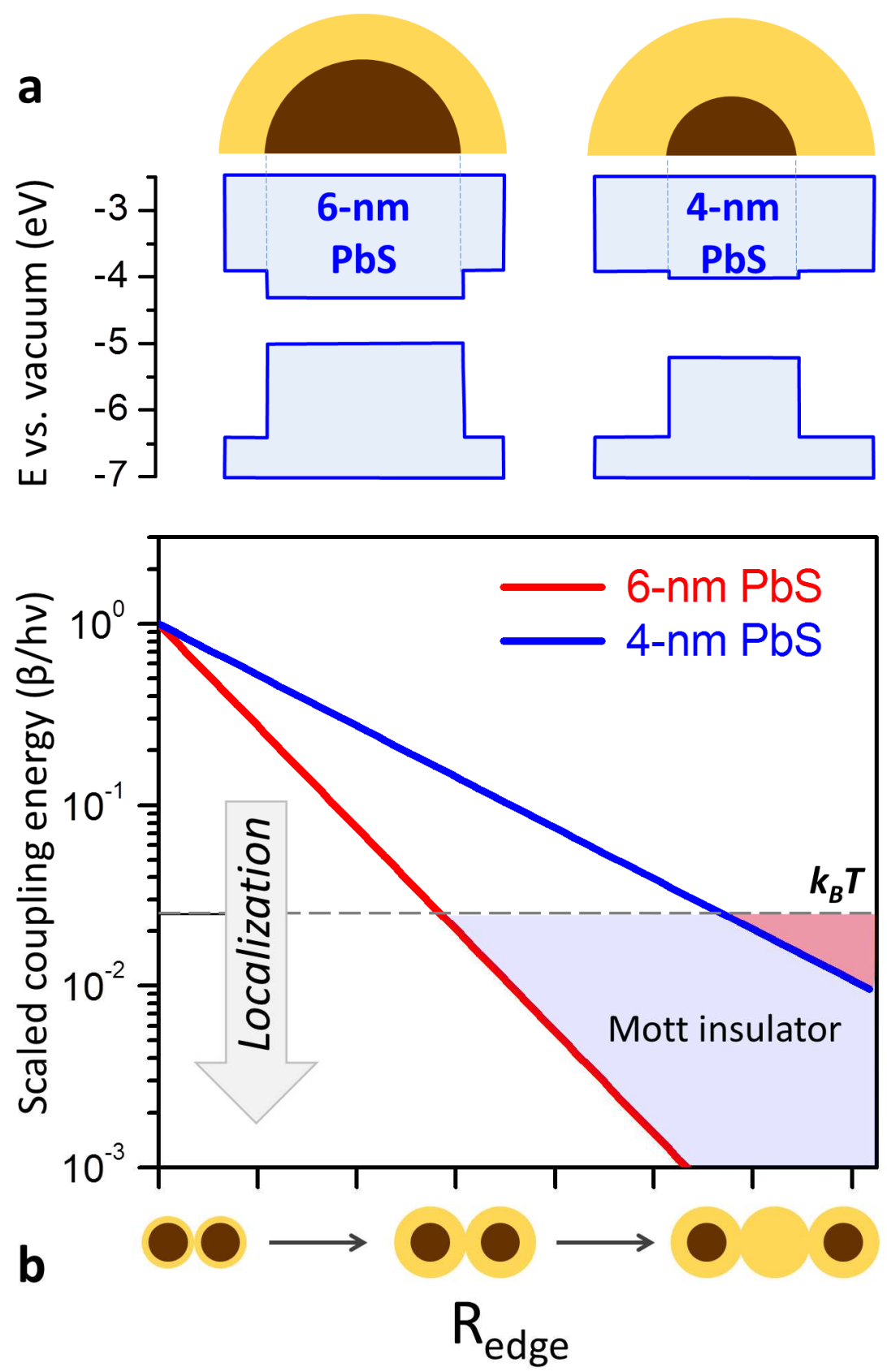

Figure 2. (a). Energy diagram showing a relative alignment of conduction and valence band edges in 4.0-nm $\mathrm{PbS} / \mathrm{CdS}$ and 6.0-nm $\mathrm{PbS} / \mathrm{CdS}$ core/shell nanocrystals. (b). WKB tunneling approximation of the coupling energy dependence on the interparticle distance $\left(\mathrm{R}_{\text {edge }}\right)$ for the two material systems shown in (a). The colored areas indicate the regime of strongly localized charges. 
According to Fig. 2b, achieving a strong localization of photoinduced charges in small-diameter $\mathrm{PbS}$ NCs requires growing a thick CdS shell needed to compensate for a shallow potential barrier to electrons in $\mathrm{PbS}$ (see Fig. 2a). A possible drawback of this strategy is the propensity of thicker shells to cause an excessive interfacial strain, which typically relaxes through the formation of defects, whereby introducing additional non-radiative decay channels. ${ }^{37-39}$ Alternatively, the width of the potential barrier separating adjacent PbS NCs could be increased by replacing a portion of PbS NCs with CdS dots of the same diameter, as illustrated in Fig. 1b. Such strategy would result in a sufficient interparticle separation needed for a strong carrier localization without the need for an additional shell growth. Within this approach, the balance between the filling factor (ff) and the emission quantum yield of embedded $\mathrm{PbS}$ NCs can be controlled by varying the ratio of $\mathrm{PbS} / \mathrm{CdS}$ to $\mathrm{CdS}$ nanoparticle precursors in the mixture. For instance, increasing the volume fraction of $\mathrm{PbS}$ NCs embedded into CdS matrices leads to the reduction of an average $\mathrm{R}_{\text {edge }}$ distance between neighboring $\mathrm{PbS}$ domains. This results in the enhancement of the filling factor and a concurrent drop in fluorescence (FL) quantum yield due to an incomplete localization of excited carriers in electrically coupled $\mathrm{PbS}$ cores. Conversely, a low volume fraction of $\mathrm{PbS}$ NCs should promote a greater emission QY due to an enhanced carrier localization but would lead to a relatively low filling factor.

To explore the effect of the interparticle separation, $\mathrm{R}_{\text {edge, }}$ on the $\mathrm{PbS}$ exciton decay, we have fabricated two sets of nanoparticle solids representing two different strategies for tuning the value of

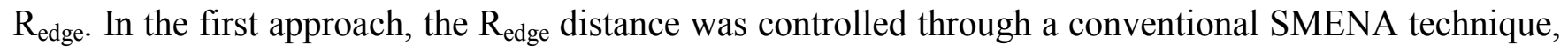
by adjusting the thickness of the $\mathrm{CdS}$ shell in $\mathrm{PbS} / \mathrm{CdS}$ core/shell nanocrystal precursors. For the second set of films, we have used a modified-SMENA strategy wherein the value of $\mathrm{R}_{\text {edge }}$ was adjusted by introducing a tunable fraction of $\mathrm{CdS}$ nanoparticles. Core/shell $\mathrm{PbS} / \mathrm{CdS} \mathrm{NCs}$ used in both sets of experiments were fabricated using a cation exchange approach. ${ }^{2}$ This method benefits from a precise control of the shell thickness, a parameter, which determines the ultimate value of $R_{\text {edge }}$ in both film 
architectures. A characteristic TEM image of core/shell $\mathrm{PbS} / \mathrm{CdS}$ NCs featuring a 2.7-nm PbS core capped with a 1.3-nm CdS shell is shown in Fig. 3a and 3b. A uniform placement of the CdS shell around the $\mathrm{PbS}$ core is evident in high resolution TEM images of PbS/CdS NCs (Fig. 3b and Fig. SF6).

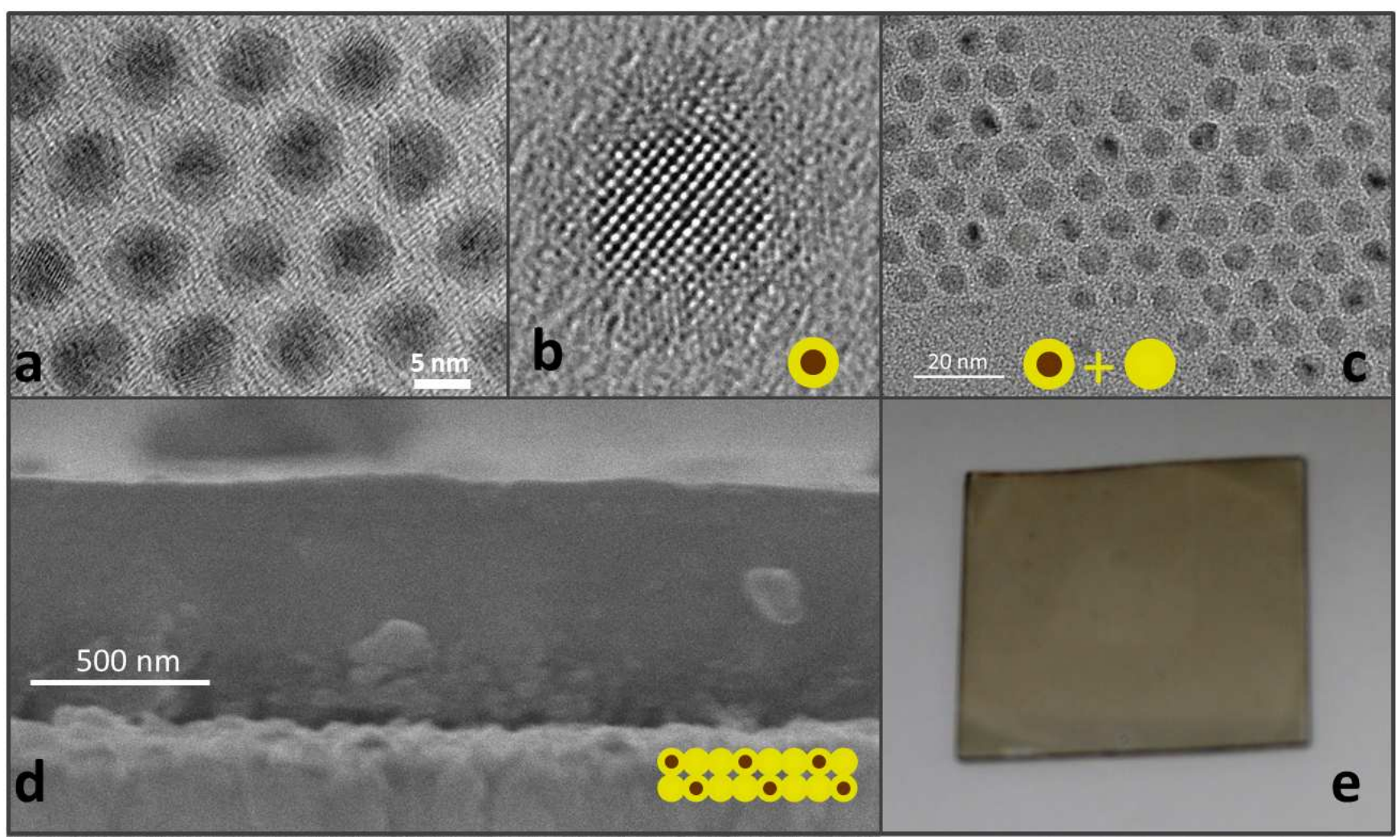

Figure 3. $(\mathrm{a}, \mathrm{b})$. High resolution TEM images of $2.7-\mathrm{nm} \mathrm{PbS}$ nanocrystals coated with a 1.3-nm CdS shell. (c). The mixture of PbS/CdS NCs and 5.0-nm CdS NCs prior to the deposition on a glass substrate. Scanning electron microscope (d) and photo (e) images of a CdS-encapsulated PbS NC film.

The fluorescence (FL) lifetime of PbS nanocrystal solids assembled using a conventional SMENA strategy (Fig. 4a) increases with the growing interparticle separation in the film, which is consistent with a diminishing probability of exciton dissociation through charge and energy transfer processes. It was previously shown that the characteristic timescale for such interparticle transfer is given by the fast component of the FL intensity decay, as illustrated in Fig SF1, while the slow component represents charge trapping on surfaces of unpassivated CdS matrices. ${ }^{40}$ The fast exponent of the film emission is therefore limited to charge and energy transfer processes that result in non-emissive states. Notably, the 
two transfer mechanisms have distinctly different $\mathrm{R}_{\text {edge }}$ dependences. According to WKB approximation, the rate of charge tunneling between resonant states decays exponentially with $\mathrm{R}_{\text {edge, }}$ while the rate of energy transfer diminishes as $\mathrm{R}^{6}$ edge in accordance with the nonradiative dipole-dipole coupling approximation (Förster resonance energy transfer). ${ }^{41}$ The comparison of experimentally measured $\tau_{\text {fast }}^{F L}$ with the WKB $\left(\sim e^{\alpha R_{E d g e}}\right)$ and FRET $\left(\sim r^{6}\right)$ model curves in Fig. $4 \mathrm{~b}$ reveals that charge transfer is a more likely mechanism to contribute into exciton dissociation. Indeed, the observed transfer times exhibit a nearly single-exponential dependence on $\mathrm{R}_{\text {edge, }}$ which is characteristic of a charge tunneling process, and not the energy transfer, which is strongly affected by small changes in $\mathrm{R}_{\text {edge. }}$ This suggests that the charge transfer between adjacent dots in the matrix is the primary mechanism responsible for the formation of non-emissive states.
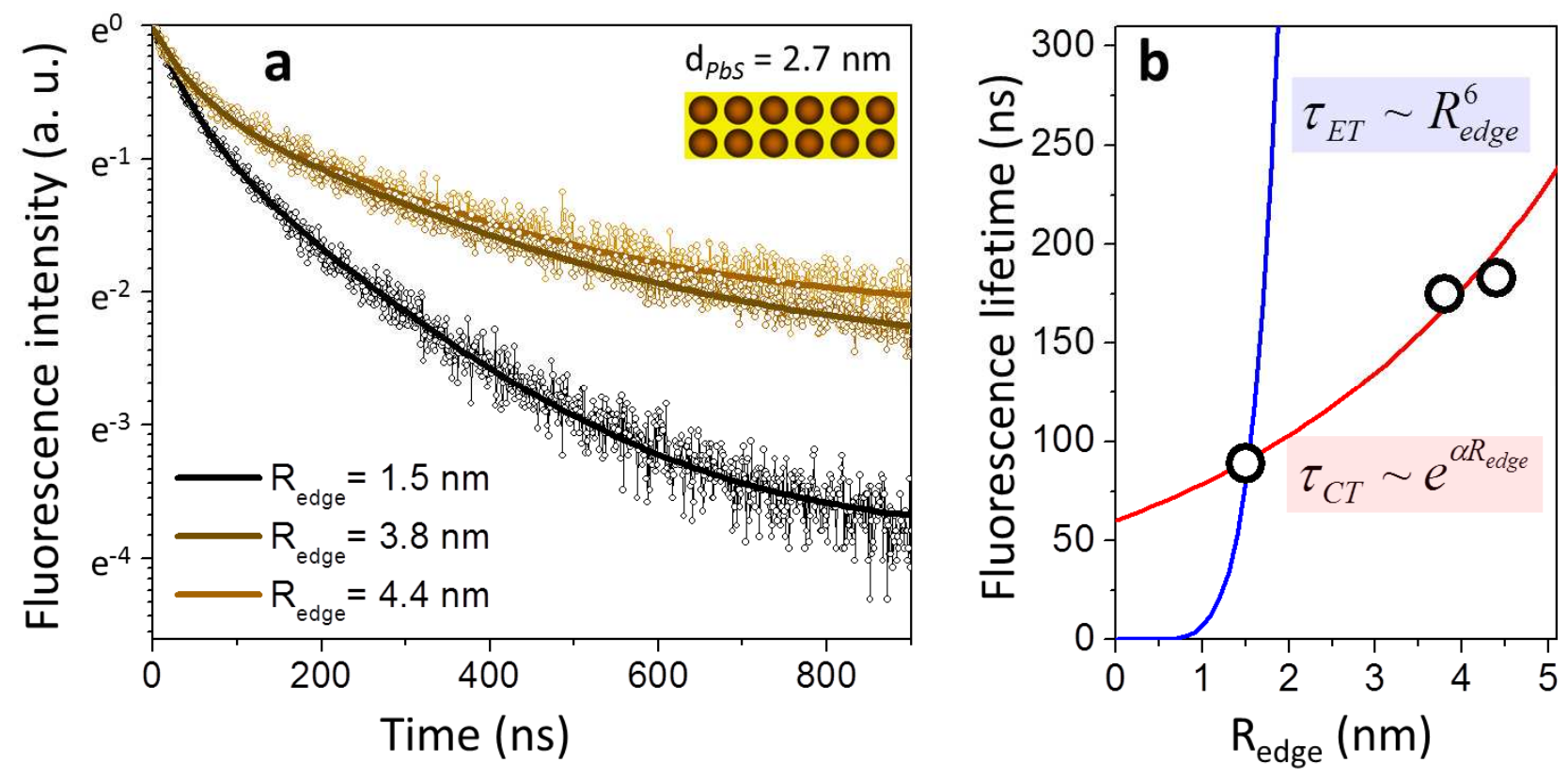

Figure 4. (a). Fluorescence intensity decay of conventional SMENA films representing different $\mathrm{R}_{\text {edge }}$ values. (b). Measured exciton lifetimes for $\mathrm{CdS}$-encapsulated $\mathrm{PbS} \mathrm{NCs}$ and their fitting by a model according to WKB (charge transfer) and dipole-dipole coupling (energy transfer) approximations.

In the case of a conventional SMENA approach, the fluorescence lifetime of matrix-encapsulated nanocrystals did not exceed $\tau=190 \mathrm{~ns}$, which is far below the 2.8 microsecond lifetime observed for the 
same nanocrystals in solution (see Fig. SF2). Even with further increases in the shell thickness beyond 3-4 monolayers, the emission lifetime remained roughly the same. Taking into account a significant suppression of the interparticle charge transfer for such large values of $\mathrm{R}_{\text {edge, }}$, the short lifetime has been tentatively attributed to a continuous build-up of the interfacial strain, manifested by the growing ratio of the trap state to band gap emission (see Fig. SF3). ${ }^{33}$ Decay of trapped carriers creates a broad band emission, which overlaps with the PbS FL peak. Subsequently, despite an enhanced exciton localization in solids featuring large interparticle distances, strong emission within a narrow spectral band cannot be reached through a conventional SMENA architecture. On the contrary, the modified SMENA approach allows enhancing the interparticle separation without the need for an epitaxial growth of large shells. By including $\mathrm{CdS}$ nanoparticles within $\mathrm{PbS} / \mathrm{CdS}$ matrices, we aim to facilitate the suppression of charge and energy transfer between adjacent $\mathrm{PbS}$ nanoparticles, while preventing the formation of rate-limiting defects.

To explore optical properties of modified-SMENA solids comprising a mixture of $\mathrm{CdS}$ and $\mathrm{PbS} / \mathrm{CdS}$ $\mathrm{NC}$ precursors, we have fabricated several films featuring an increasing fraction of $\mathrm{CdS}$ to $\mathrm{PbS}$ nanoparticles. The volume fraction of PbS NCs in these solids was calculated based on the known ratio of $\mathrm{PbS}$ to $\mathrm{CdS}$ particle absorbance. Namely, when $\mathrm{CdS}$ and $\mathrm{PbS} / \mathrm{CdS}$ core/shell colloids are fused into a film, the average interparticle distance, $\mathrm{R}_{\text {edge, }}$, becomes greater than twice the thickness of the CdS shell as the distance between the $\mathrm{PbS}$ dots is now enhanced by the presence of interstitial $\mathrm{CdS}$ domains. To determine the relationship between molar concentrations of both nanoparticle types and the partial number density of these nanocrystals in a mixture, precursor solutions of mixed nanoparticles were deposited onto a TEM grid and analyzed. According to Fig. 5e, the ratio of each nanoparticle type ( $\mathrm{PbS} / \mathrm{CdS}$ core/shell versus $\mathrm{CdS}$ dot) can be easily obtained by identifying the corresponding populations of each dot in the TEM specimen. The core/shell nanocrystals can be differentiated by their larger radii and darker shading, as indicated by arrows in a characteristic TEM image (Fig. 5e), featuring 4.3-nm CdS and 5.2-nm PbS/CdS NCs. Since the initial concentrations of each colloid prior to TEM sampling were known, the number density of each nanocrystal type in a film can be estimated. The ratio 
of $\mathrm{CdS}$ to $\mathrm{PbS} / \mathrm{CdS}$ dots in the film is then used to estimate the $\mathrm{PbS}$ volume fraction, $v_{\mathrm{PbS}}$, according to the following equation (see Supporting Information for details of calculation):

$$
v_{P b S}=\frac{(n+1)^{2} \times 4 / 3 \pi R_{\text {core }}^{3}}{\left(n D_{C}+D_{P / C}\right)^{3}}
$$

where $n=N_{P b S} / N_{C d S}$, and $R_{\text {core }}$ is the average radius of the $\mathrm{PbS}$ core domain in $\mathrm{PbS} / \mathrm{CdS}$ core/shell NCs.
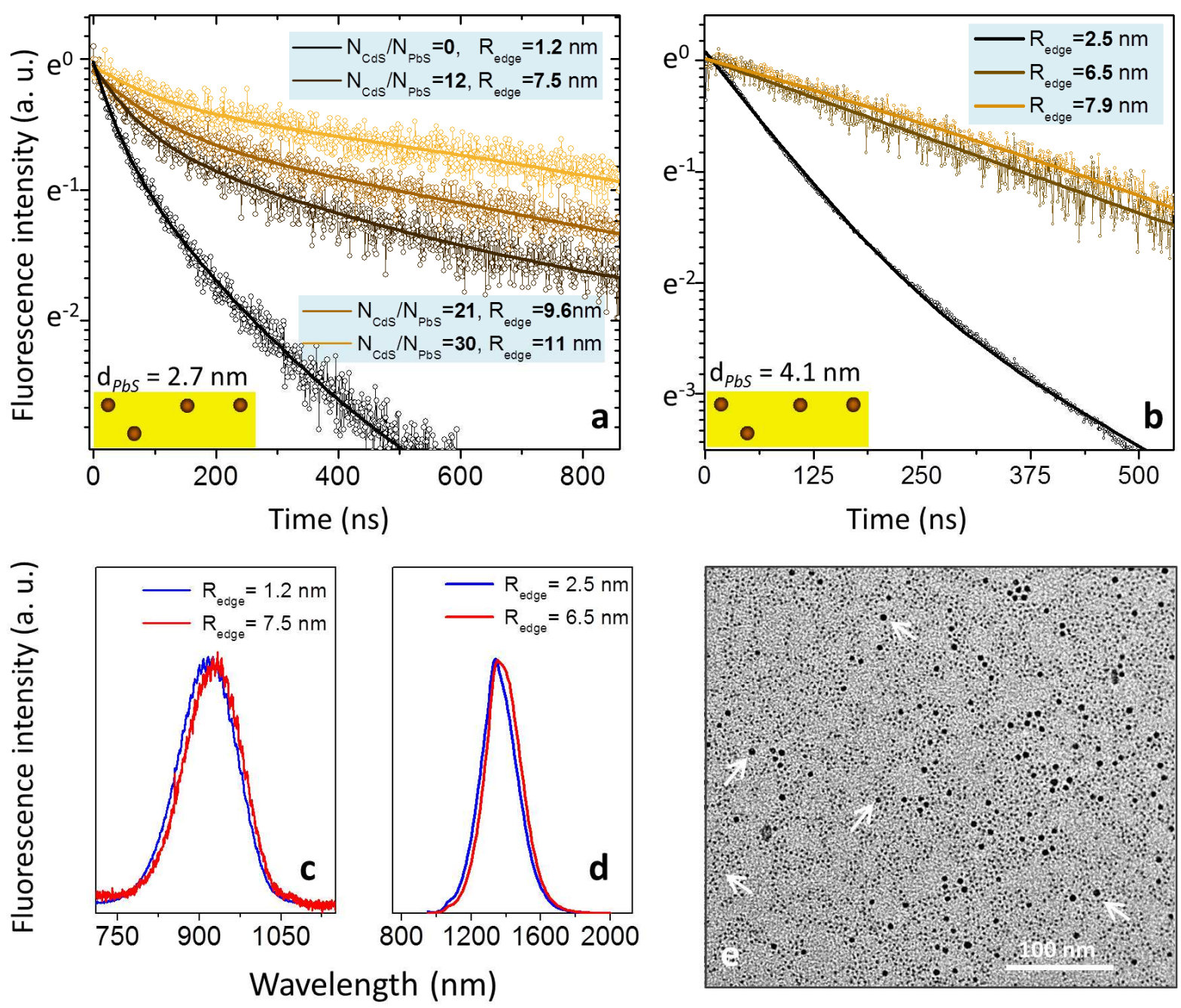

Figure 5. Fluorescence intensity decay of near-IR emitting PbS NCs (a) $d=2.7 \mathrm{~nm}$ and (b) $d=4.1$ $\mathrm{nm}$ encapsulated into CdS matrices as a function of increasing $N_{C d S} N_{P b S}$ "precursor nanoparticle" fraction. (c). Steady-state emission of modified-SMENA films $\left(\mathrm{d}_{\mathrm{PbS}}=2.7 \mathrm{~nm}\right)$ featuring $N_{C d S} N_{P b S}=0$ (blue curve) and $N_{C d S}^{\prime} N_{P b S}=12$ (red curve). (d). Steady-state emission of modified-SMENA films $\left(\mathrm{d}_{\mathrm{PbS}}=\right.$ 
$4.1 \mathrm{~nm}$ ) representing $N_{C d S} N_{P b S}=0$ (blue curve) and $N_{C d S}^{\prime} N_{P b S}=4$ (red curve). (e). A characteristic TEM image of $4.3-\mathrm{nm} \mathrm{CdS}$ and $6.0-\mathrm{nm} \mathrm{PbS} / \mathrm{CdS} \mathrm{NC}$ mixture. Arrows mark the location of $\mathrm{PbS} / \mathrm{CdS}$ core/shell structures.

Figure 5a shows the FL intensity decay of mixed $\mathrm{CdS}\left(\mathrm{d}_{\mathrm{CdS}}=4.3 \mathrm{~nm}\right)$ and $\mathrm{CdS} / \mathrm{PbS}\left(\mathrm{d}_{\mathrm{PbS}}=2.7 \mathrm{~nm}\right)$ NC solids fabricated using a modified SMENA approach. As expected from WKB approximation, the FL lifetime increases with growing $\mathrm{R}_{\text {edge, }}$ reflecting a simultaneous decrease in the cumulative rate of interparticle transfer processes. The fastest decay in Fig. 5a $\left(\mathrm{R}_{\text {edge }}=1.2 \mathrm{~nm}\right)$ corresponds to a solid containing no CdS NCs (conventional SMENA). A relatively thin CdS shell $(\Delta \mathrm{H} \approx 1 \mathrm{~nm})$ used in this case enabled only a partial localization of charges, leading to a charge transfer-limited lifetime of about 95 ns. The addition of $\mathrm{CdS} \mathrm{NCs}$ to $\mathrm{PbS} / \mathrm{CdS}$ precursor nanoparticles increases the interparticle separation from 1.2 to $11 \mathrm{~nm}$ (Fig. 5a), bringing the maximum $\mathrm{R}_{\text {edge }}$ to more than twice the value accessible through a conventional SMENA approach. The enhanced interparticle separation results in a stronger localization of photoinduced charges (as illustrated in Fig. 2b), which causes the FL lifetime to increase (Fig 5a). A particularly interesting trend was observed for large values of $\mathrm{R}_{\text {edge. }}$ For these matrices, the lifetime of $\mathrm{PbS}$ excitons climbed to an unprecedented $0.98 \mu$ s (determined by a $1 / \mathrm{e}$ intensity drop), which constitutes a four-fold increase over best-performing NC emitters fabricated through a conventional SMENA approach. We note that the exciton lifetime for these nanocrystals in solution is $2.8 \mu \mathrm{s}$ (Fig. SF2), which implies that a substantial fraction of excitations recombine radiatively, setting the upper limit of the FL QY to nearly $100 * \tau_{\mathrm{FL}} / \tau_{\text {radiative }}=10 \%$ (although absolute QY was not measured for these samples). Likewise, the observed exciton lifetime was found to be enhanced relative to those of previously reported $\mathrm{PbS} \mathrm{NC}$ film architectures utilizing hybrid molecular ligands. ${ }^{2}$ This phenomenon can be tentatively attributed to a suppressed non-radiative decay in matrixpassivated nanocrystals. The saturation of the fluorescence lifetime in modified-SMENA films emitting at $\lambda \approx 1300 \mathrm{~nm}$ occurs faster than in small-diameter PbS NC films (see Fig. 5b), with the longest lifetime 
of $\tau=480 \mathrm{~ns}$. An enhanced lifetime of band gap excitons in mixed PbS NC films is consistent with the reduced volume fraction of $\mathrm{PbS} \mathrm{NCs}$ in comparison with conventional SMENA solids. Finally, according to Figs. 5c,d, the increase in $\mathrm{R}_{\text {edge }}$ value gives rise to a $<20 \mathrm{~nm}$ redshift of the emission peak, as expected due to the delocalization of the electronic wave functions into the CdS matrix.

An absolute value of the emission quantum yield was determined for films comprising largediameter $\operatorname{PbS} \operatorname{NCs}\left(\lambda_{\mathrm{FL}} \approx 1300 \mathrm{~nm}\right)$. We note that these films did not yield the best exciton lifetime, however, were more compatible with the experimental setup in use. The detection methodology was adapted from the one described in Ref. 45, and was designed to eliminate the use of a reference dye and to provide the possibility for simultaneous measurement at excitation wavelength of absorbance, which is corrected to reflectance and scattering losses at integrating sphere. According to these measurements (summarized in Table ST1), the highest value of the emission quantum yield of QY=3.7\% was realized for solids featuring $\mathrm{R}_{\text {edge }}$ of $7.9 \mathrm{~nm}$. To the best of our knowledge, this value represents the highest reported emission QY for inorganic nanocrystal films emitting in the infrared range. The difference between the observed QY value in a solid and a typical QY range for these nanoparticles in solution (10$30 \%$ ) is attributed to the onset on non-radiative decay pathways (trapping and charge transfer) upon the transfer of colloidal nanoparticles onto a substrate.

Another relevant property of modified SMENA films that merits discussion is the linewidth of the emission peak. Application of luminescent films in light-emitting technologies often benefits from a spectrally narrow emission profile. Our estimates show that the peak width of modified-SMENA films $(\mathrm{FHWM}=165 \mathrm{~nm}, @ \lambda=970 \mathrm{~nm}$ ) increases compared to that of OA-capped $\mathrm{PbS} \mathrm{NC}$ in solution $($ FHWM $=120 \mathrm{~nm}, @ \lambda=950 \mathrm{~nm})$. However, the fusion of core/shell NCs into a solid doesn't not seem to contribute much to broadening of the emission profile, as OA-capped $\mathrm{PbS} / \mathrm{CdS} \mathrm{NCs}$ in solution exhibit FHWM of $160 \mathrm{~nm}$. Indeed, the primary enhancement of the peak width occurs as a result of the shell growth, an important step, which also results in the improvement of the FL QY. 


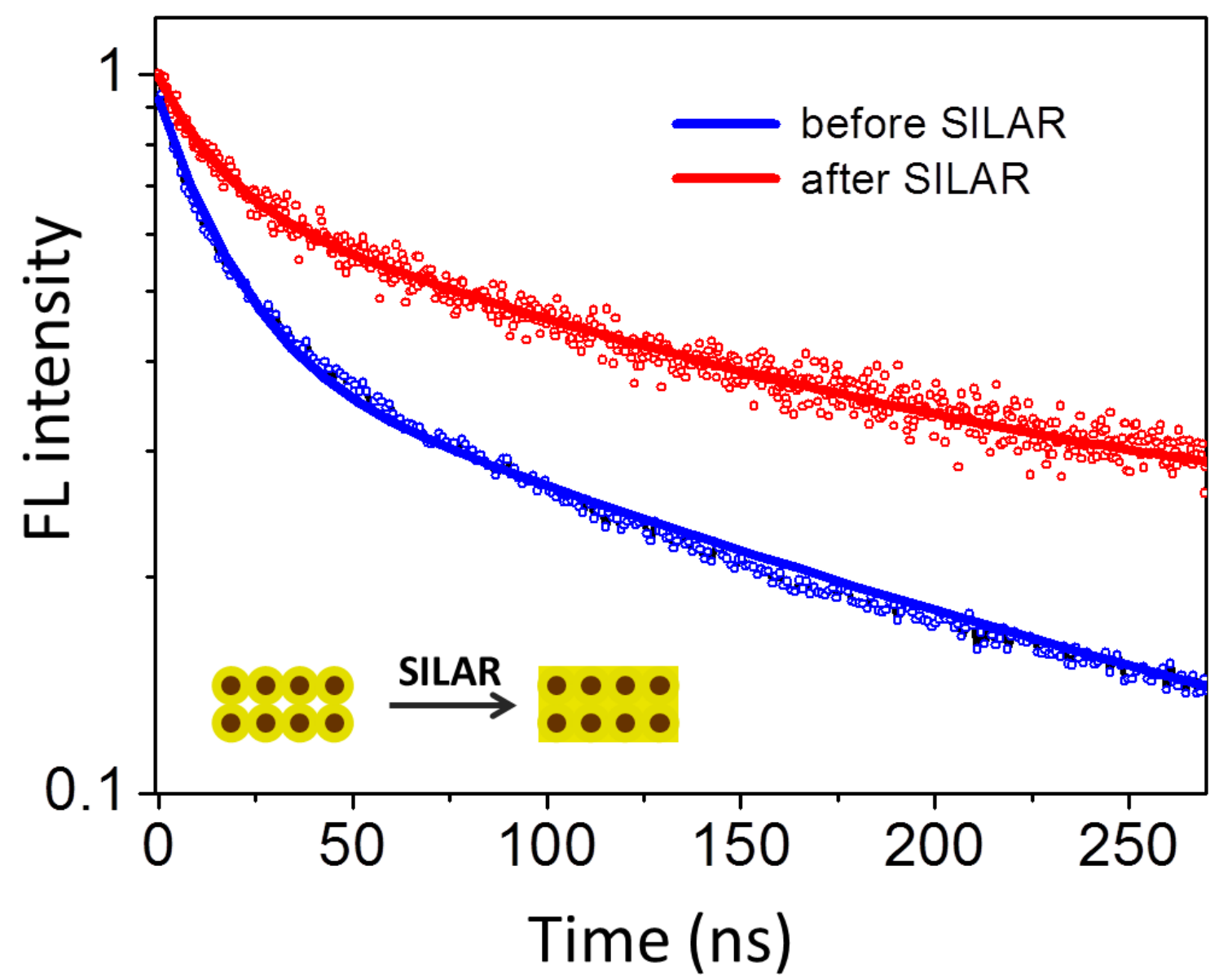

Figure 6. Suppression of charge trapping on the surface of $\mathrm{PbS} / \mathrm{CdS}$ SMENA films $\left(\mathrm{R}_{\mathrm{edge}}=1.5 \mathrm{~nm}\right.$, $\mathrm{d}_{\mathrm{PbS}}=2.7 \mathrm{~nm}$ ) by additional SILAR passivation. The red curve represents an increased exciton lifetime following the SILAR treatment.

In order to reduce the density of dangling bonds in nanocrystal matrices, interparticle gaps between fused core/shell structures were partly filled with additional CdS. To this end, several SILAR cycles were applied resulting in a deposition of 3-4 CdS monolayers. Previous works have shown ${ }^{34}$ that such treatment increases the refractive index of the nanocrystal film through partial filling of void areas. According to Fig. 6, application of 6 SILAR cycles to a CdS-encapsulated PbS NC film featuring $\mathrm{R}_{\text {edge }}$ of $1.5 \mathrm{~nm}$ results in a 55\% increase in the FL lifetime. In particular, the slower component of the FL 
intensity decay, which is associated with charge tapping has increased from $\tau_{F L \text {, slow }}=90$ to $\tau_{F L \text {, slow }}=150$ ns, reflecting the reduction in the rate of carrier trapping.

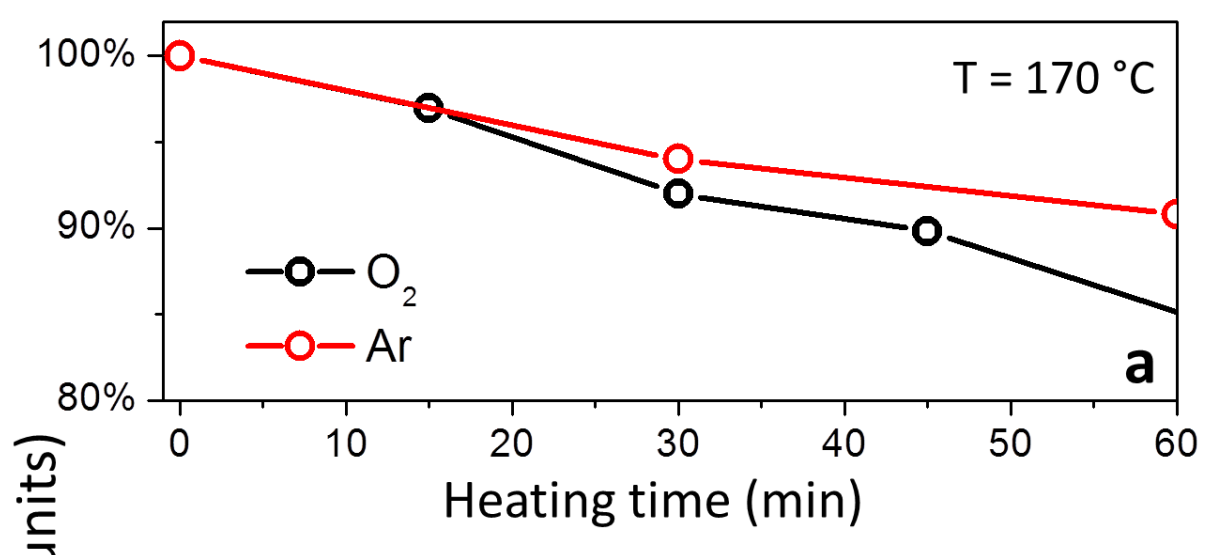

CdS-encapsulated PbS films

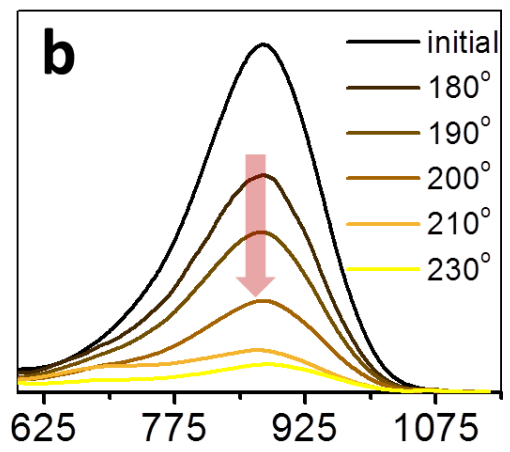

CdS-encapsulated PbS films

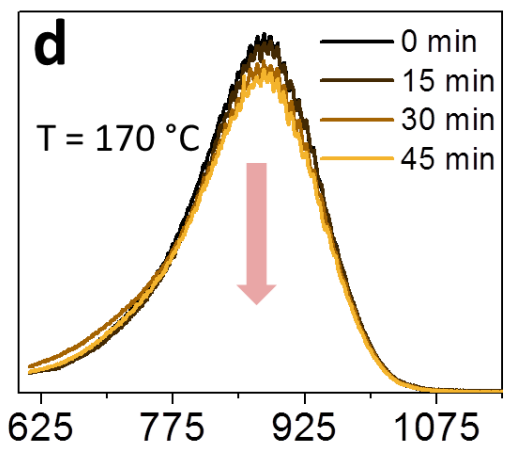

Wavelength $(\mathrm{nm})$
OA-capped PbS films

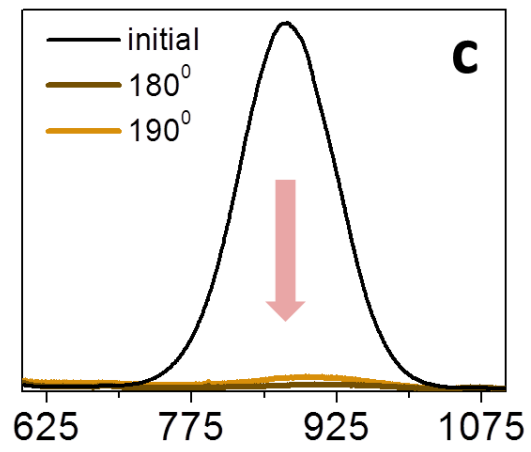

OA-capped $\mathrm{PbS} / \mathrm{CdS}$ core/shell

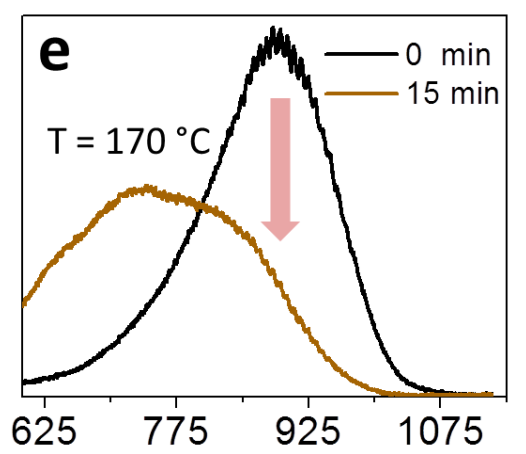


thermal stability of OA-capped (c) and CdS-encapsulated (b) PbS NC arrays, fabricated using a modified-SMENA approach. $(\mathrm{d}, \mathrm{e})$. Temporal evolution of the emission peak for films expoosed to $\mathrm{T}=170{ }^{\circ} \mathrm{C}$ in air. The comparison is drawn between CdS-encapsulated $\mathrm{PbS} \mathrm{NC}$ arrays (d) and OAcapped $\mathrm{PbS} / \mathrm{CdS}$ core/shell NCs on a substrate (e).

Improving the thermal stability of NC solids is important for their deployment in light-emitting devices, as many of those applications are subject to the generation of heat. In the present approach, the presence of heteroepitaxial bonding between $\mathrm{NC}$ surfaces and the matrix semiconductor is expected to prevent low-temperature oxidation, deferring the thermal degradation of NCs to occur only at higher temperatures via the phase transition of the lattice. Here, we test this hypothesis by comparing thermal stability of modified-SMENA films $\left(\mathrm{R}_{\mathrm{edge}}=2.8 \mathrm{~nm}\right.$, SILAR treated $)$ with that of oleic acid (OA)-capped PbS NC films. To this end, the intensity of band edge emission in both solids was plotted versus the substrate temperature. According to Figs. 7b,c, heating of matrix-encapsulated (b) and OA-passivated (c) $\mathrm{PbS}$ NCs in air results in a gradual drop of the emission intensity, which was taken as a sintering temperature of $\mathrm{PbS}$. In the case of CdS-encapsulated $\mathrm{PbS} \mathrm{NCs}$, sintering occurred at $\mathrm{T} \approx 200{ }^{\circ} \mathrm{C}$, which is $90-100{ }^{\circ} \mathrm{C}$ above that of OA-capped $\mathrm{PbS} \mathrm{NC}$ solids. Interestingly, the thermal stability of modifiedSMENA PbS solids does not arise merely from the presence of the $\mathrm{CdS}$ shell on $\mathrm{PbS}$ surfaces. According to Figs. 7d,e, oleic acid-capped $\mathrm{PbS} / \mathrm{CdS} \mathrm{NCs}\left(\Delta \mathrm{H}_{\mathrm{CdS}}=1.2 \mathrm{~nm}\right)$ placed on a hot substrate undergo a much faster degradation of the band gap emission then modified SMENA films fabricated from the same core/shell nanoparticles. This result strongly suggests that the crystallographic fusion of core/shell precursors into a solid is essential for enhancing the stability of encapsulated PbS NCs. In the final stability test, we have looked into the temporal degradation of the film versus time by recording the 
changes in the emission intensity after the heat was applied to the substrate. The two curves in Fig. 7a, illustrate the reduction in the emission intensity of matrix-encapsulated $\mathrm{PbS} \mathrm{NCs}$ for films kept in argon (red) and air (blue). A slightly faster decay of the latter curve suggests that oxidation is partly responsible for the loss of emission at elevated temperatures. These experiments demonstrate an enhanced heat resistance of matrix-encapsulated $\mathrm{PbS}$ SMENA solids as compared to organic-interlinked nanoparticle films.

\section{Conclusions.}

In conclusion, a novel strategy for processing of $\mathrm{PbS}$ nanocrystal solids exhibiting bright infrared emission is demonstrated. The reported methodology benefits from the use of inorganic CdS host matrices to provide a stable passivation of nanoparticle surfaces while preserving some degree of quantum confinement. The morphology of encapsulating matrices was judiciously optimized to induce the suppression of non-radiative decay processes associated with surface carrier trapping and interparticle charge transfer. This allowed extending exciton lifetimes up to 1 microsecond, which constitutes at least a two-fold enhancement over ligand-based passivation strategy. The suppressed carrier trapping has given rise to a high emission quantum yield of $\mathrm{QY}_{\text {solid }}=3.7 \%$ in the infrared range, while inorganic matrix medium warranted a superior thermal and chemical stability of fabricated nanocrystal solids.

\section{Experimental Section.}

Materials. 1-octadecene (ODE, 90\% Aldrich), oleic acid (OA, 90\% Aldrich), lead(II) oxide powder ( $\mathrm{PbO}$, 99.999\% Aldrich), cadmium oxide (CdO, 99.99\%, Aldrich), sodium sulfide nonanhydrate $\left(\mathrm{Na}_{2} \mathrm{~S} \cdot 9 \mathrm{H}_{2} \mathrm{O}, 98 \%\right.$ Alfa Aesar), sulfur (S, 99.999\% Acros), ethanol (anhydrous, 95\% Aldrich), methanol (anhydrous, 99.8\% Aldrich), toluene (anhydrous, 99.8\% Aldrich), isopropanol (anhydrous, 99.8\% Acros), octane (anhydrous, 99\% Aldrich), hexane (anhydrous, 95\% Aldrich), 3-mercaptopropionic acid 
(3-MPA, 99\% Alfa Aesar), bis(trimethylsilyl) sulfide ( $\mathrm{TMS}_{2}$, Aldrich, synthetic grade), chloroform (anhydrous, 99\%, Aldrich), cadmium acetate dihydrate $\left(\left(\mathrm{CH}_{3} \mathrm{COO}\right)_{2} \mathrm{Cd} \cdot 2 \mathrm{H}_{2} \mathrm{O}, 98 \%\right.$, Acros), zinc acetate (98+\% Acros), acetone (anhydrous, Amresco, ACS grade) were used as received without any further purification. All reactions were performed under argon atmosphere using the standard Schlenk technique. Glass substrates (Corning Microscope Slides, 0.96-1.06 mm, 0215 Glass) were obtained from Corning Inc.

\section{Methods.}

Fabrication of PbS nanocrystals. $\mathrm{PbS} \mathrm{NCs}$ were fabricated according to a procedure adapted from Hines et l. $^{42}$ In a typical synthesis, a mixture of $0.49 \mathrm{~g}(2 \mathrm{mmol}) \mathrm{PbO}$ in $18 \mathrm{~mL}$ of ODE and 1-16 mL of OA (increasing the amount of OA results in larger NCs) was degassed in a three-neck flask at $120^{\circ} \mathrm{C}$ for 2 hours, switched to Argon, and heated to $135^{\circ} \mathrm{C}$. Meanwhile, $10 \mathrm{~mL}$ of ODE was degassed for two hours at $120^{\circ} \mathrm{C}$ and allowed to cool down to room temperature. Then, $0.21 \mathrm{ml}$ of (TMS) $)_{2} \mathrm{~S}$ was added carefully into the flask and the resulting (TMS) $)_{2} \mathrm{~S} / \mathrm{ODE}$ mixture was injected into the $\mathrm{Pb}$ precursor solution at $135^{\circ} \mathrm{C}$, while stirring. The reaction was stopped after 0-5 minutes (longer reaction time leads to larger NCs) by removing the flask from the heating mantle and placing it into an ice water bath. The nanocrystals were isolated from the mixture by precipitating with acetone, centrifuging, and redispersing in toluene. The cleaning procedure was repeated 2-3 times, after which nanocrystals were redispersed in a minimal amount of hexane (4-5 ml).

For example, to fabricate PbS NCs with an exciton absorption feature centered at $1050 \mathrm{~nm}\left(\mathrm{~d}_{\mathrm{PbS}}=\right.$ $3.5 \mathrm{~nm}$ ), $2 \mathrm{~mL}$ of OA was used and the growth time was limited to 1 min. For NCs with a $1 \mathrm{~S}$ peak at $1300 \mathrm{~nm}\left(\mathrm{~d}_{\mathrm{PbS}}=4.6 \mathrm{~nm}\right), 14 \mathrm{~mL}$ of OA was used with the growth time of $4 \mathrm{~min}$. For NCs with a $1 \mathrm{~S}$ peak at $930 \mathrm{~nm}\left(\mathrm{~d}_{\mathrm{PbS}}=3.0 \mathrm{~nm}\right), 1.5 \mathrm{~mL} \mathrm{OA}$ was used with the reaction time of $1 \mathrm{~min}$.

Synthesis of $\mathbf{P b S} / \mathbf{C d S}$ core/shell NCs. The growth of the CdS shell on PbS NCs was performed using a cation exchange methodology. ${ }^{2}$ To this end, $0.95 \mathrm{~g}(7.6 \mathrm{mmol})$ of $\mathrm{CdO}$ was dissolved in $10 \mathrm{ml}$ of $\mathrm{OA}$ and $18 \mathrm{ml}$ of ODE under Ar at $235^{\circ} \mathrm{C}$ until the solution became clear. Then, the temperature of this solution was set at $130^{\circ} \mathrm{C}$. Meanwhile, $4 \mathrm{ml}$ of $\mathrm{PbS}$ seed nanocrystals $(\mathrm{d}=4.2 \mathrm{~nm})$ solution in hexane (concentration $10 \mathrm{mg} / \mathrm{ml}$ ) was kept under argon gas flow at $80^{\circ} \mathrm{C}$ for 2 minutes to remove excess of solvent and then was injected into the cadmium solution under vigorous stirring. The reaction mixture was kept at $135{ }^{\circ} \mathrm{C}$ for 7 minute and then stopped by immersing the flask in the ice water bath. The purification process included the separation of nanoparticles from the solution with $\sim 30 \mathrm{ml}$ of ethanol, 
centrifugation and dissolution of the precipitated nanocrystals in a minimal amount of hexane. The cleaning cycle was repeated two times. Under these conditions the absorbance peak was found to blueshift by $330 \mathrm{~nm}$ (approx. $0.8 \mathrm{~nm}$ of the CdS shell). The growth of the CdS shell depended on the size $\mathrm{PbS} \mathrm{NCs}$ and the reaction time. Longer reaction times, higher temperatures and higher ratios of OA/ODE were used to obtain a thicker shell of CdS.

To determine the shell thickness in thin-shell nanocrystals (where TEM images do not provide the sufficient accuracy) the following approach was used. First, the size of original PbS dots was determined from the position of the exciton absorption edge. The reduction in the average size of the $\mathrm{PbS}$ domain upon $\mathrm{Pb}^{2+}$ to $\mathrm{Cd}^{2+}$ cation exchange was determined from the new position of the $\mathrm{PbS}$ exciton peak. The thickness of the shell was then calculated under the assumption that the diameter of the $\mathrm{PbS} / \mathrm{CdS}$ core/shell structure remained the same as the diameter of the original $\mathrm{PbS}$ dot.

Synthesis of CdS nanoparticles. For the fabrication of CdS seeds an approach proposed by $\mathrm{Yu}$ et al. was used. ${ }^{43}$ The mixture of $0.0384 \mathrm{~g}$ ( $\left.0.3 \mathrm{mmol}\right) \mathrm{CdO}, 1 \mathrm{ml} \mathrm{OA}$ and $11 \mathrm{ml} \mathrm{ODE}$ in a $50 \mathrm{ml}$ 3-neck flask was heated to $300^{\circ} \mathrm{C}$ until solution turned optically clear and colorless. Then, the temperature of the reaction mixture was set up at $250^{\circ} \mathrm{C}$ and a sulfur precursor solution made by dissolving sulfur powder $0.0048 \mathrm{~g}(0.15 \mathrm{mmol})$ at $200^{\circ} \mathrm{C}$ in $\mathrm{ODE}(4.5 \mathrm{~mL})$ was quickly injected. The reaction was stopped by removing the flask from the heating mantle after 5-9 minutes. CdS NCs were separated from the solution by precipitation with methanol followed by repeated hexane/methanol extractions. The final nanocrystals had diameter 3.5-4.5 $\mathrm{nm}$ (depending on the reaction time) and were stored in hexane.

Preparation of the glass substrate. Before the deposition of nanocrystals the glass substrates were cleaned and dry for better performance. To this end, glass squares $2.5 \mathrm{~cm}$ x $2.5 \mathrm{~cm}$ were washed with detergent (Alconox) and rinsed in deionized water. Subsequently, substrates were sonicated in methanol, acetone and isopropanol for 5 minutes in each solvent and dry under argon flow.

Fabrication of nanocrystal solids. The fabrication of all-inorganic NC films was performed using previously reported Semiconductor Matrix Encapsulated Nanocrystal Array (SMENA) methodology. ${ }^{44}$

To deposit a layer of all-inorganic CdS-encapsulated NC film onto the FTO/glass substrate, 3-5 drops of the $\mathrm{PbS} / \mathrm{CdS}$ core $/ \mathrm{shell} \mathrm{NC}$ solution in hexane (concentration $10 \mathrm{mg} / \mathrm{ml}$ ) were placed onto the spinning at $3000 \mathrm{rpm}$ glass until the surface was covered and then dry. To replace original OA ligands with thermally-degradable MPA molecules, 7-10 drops of MPA/Methanol solution (ratio1:4) were deposited on the center of the glass slide, soaked for 10 seconds and spun at $3000 \mathrm{rpm}$ for 10 seconds. 
After the MPA treatment, the film was washed by covering with 10 drops of methanol and spinning the slide for 10 seconds, followed by rinsing with octane in the same manner. Upon the deposition of 2 layers the films were annealed at $120-140^{\circ} \mathrm{C}$ for 15 mins. The total of 4-6 layers were deposited. To fabricate modified SMENA with additional CdS NCs, the initial solution of PbS/CdS NCs was mixed with the CdS solution in ratios, which were calculated by dividing the optical densities at the $1^{\text {st }}$ excitonic peak for $\mathrm{CdS}(\lambda=430 \mathrm{~nm})$ and PbS/CdS NCs $(\lambda \approx 850-900 \mathrm{~nm})$ colloidal precursors. The final volume ratios of nanocrystals were calculated using TEM data from utilized solutions, as discussed in the text.

In-filling of SMENA pores with CdS. For the pore-filling process, the successive ionic layer adsorption and reaction (SILAR) method was applied. ${ }^{36}$ Briefly, the deposition of additional layers of CdS was conducted by sequential soaking of the annealed NC films in methanol solutions of Cd and S precursors. For this purpose, the cadmium bath was prepared by dissolution of $0.113 \mathrm{~g}$ of cadmium acetate in $20 \mathrm{~mL}$ of methanol and the sulfur bath by placing $0.098 \mathrm{~g}$ of $\mathrm{Na}_{2} \mathrm{~S} \cdot 9 \mathrm{H}_{2} 0$ in $20 \mathrm{~mL}$ of methanol. One SILAR cycle included soaking of the film in the cadmium bath for 1 minute, then rinsing the film with methanol for 1 minute, then soaking it in the sulfur bath for 1 minute with sequential washing in methanol. 2-10 cycles of pores filling process was applied for all-inorganic films followed by annealing at $150^{\circ} \mathrm{C}$ for 15 minutes.

Characterization. Absorbance spectra were recorded using CARY 5000 and Shimadzu UV-3600 UV-vis-NIR spectrophotometers. Photoluminescence spectra were recorded using a Jobin Yvon Fluorolog FL3-11 fluorescence spectrophotometer and using a home-built photoluminescence spectroscopy apparatus including an Argon ion laser Reliant 150M (Laser Physics), an Acton SP-2357 Monochromator (Princeton Instrument) and an IR detector PDA30G (Thorlabs). High-resolution transmission electron microscopy (HR-TEM) measurements were carried out using JEOL 3011UHR and 2010 transmission electron microscopes, operated at 300 and $200 \mathrm{kV}$, respectively. To prepare a TEM sample, a small amount of NC film was scraped, dispersed in toluene by sonication, dropped onto a carbon-coated copper grid, and allowed to dry in air. FL lifetime measurements in 900nm region were performed using a time-correlated single photon counting setup utilizing SPC-630 single-photon counting PCI card (Becker \& Hickle $\mathrm{GmbH}$ ), picosecond diode laser operating at $400 \mathrm{~nm}$, as an excitation source (Picoquant), an id50 avalanche photodiode (Quantique), and long-pass optical filters with edges at 400nm, 532nm, 750nm and 900nm. Time - resolved PL traces in $1300 \mathrm{~nm}$ region were 
obtained by Time-Correlated Single Photon Counting (TCSPC) system based on InGaAs TE cooled single photon avalanche photodiode(ID Quantique) of 200 ps time resolution, adjusted for 10\% quantum efficiency, SPC-130-EM Counting Module and BDL-488-SMN laser (Becker \& Hickle) with pulse duration of $50 \mathrm{ps}$ and wavelength of $488 \mathrm{~nm}, \mathrm{CW}$ power equivalent of about $0.5 \mathrm{~mW}$, externally triggered at $1 \mathrm{MHz}$ repetition rate. PL emission from the samples passed through long-pass optical filters with edges at $500 \mathrm{~nm} 1300 \mathrm{~nm}$ in order to reject excitation laser line and possible emission from CdS matrix.

Fluorescence quantum yield measurements. The absolute value of quantum yield was measured by method, similar to the one described in Ref. 45 . In our case the advantage was the elimination of a reference and a possibility of simultaneous measurement of absorbance at excitation wavelength, with correction to reflectance and scattering losses using integrating sphere. As excitation source CW Laser Diode Module with wavelength of $808 \mathrm{~nm}$ with power of $1 \mathrm{~W}$ modulated by optical chopper at $30 \mathrm{~Hz}$, was used. For spatial averaging the integrating sphere IS200-4, (Thorlabs) was applied. This light was measured by broadband $(0.1-20 \mu \mathrm{m})$ UM9B-BL-DA pyroelectric photodetector(Gentec-EO). The modulated signal from the detector was recovered by lock-in amplifier SR 830 (Stanford Research). The signal coming out from the integrating sphere was attenuated with neutral density filter in order to set the light intensity to the optimum of dynamic range for detector and lock-in amplifier. The ratio between emitted and absorbed light gives an energy yield. This value than is transformed to the quantum yield taking into the account difference in photon energies for laser and PL band (for PL band was used average energy). The quantum yield value was corrected by transmission of applied edge-pass filters.

Acknowledgment. We gratefully acknowledge OBOR "Material Networks" program and NSF Awards CHE-1112227, and CBET-1236355 for financial support. PM was supported by a McMaster Fellowship. M.K. and S.Y. acknowledge the partial financial support from the European Union through the FP7 (ERC Starting Grant NANOSOLID, GA No.306733). 
Supporting Information Available. Experimental details, additional TEM and HRTEM images. This material is available free of charge via the Internet at http://pubs.acs.org.

\section{References.}

${ }^{1}$ van Veggel, F. C. J. M. Near-Infrared Quantum Dots and Their Delicate Synthesis, Challenging Characterization, and Exciting Potential Applications. Chem. Mater. 2014, 26, 111-122.

2 Kovalenko, M. V.; Schaller, R. D.; Jarzab, D.; Loi, M. A.; Talapin, D. V. Inorganically Functionalized $\mathrm{PbS}-\mathrm{CdS}$ Colloidal Nanocrystals: Integration into Amorphous Chalcogenide Glass and Luminescent Properties. J. Am. Chem.Soc. 2012, 134, 2457-2460.

${ }^{3}$ Pietryga, J. M.; Werder, D. J.; Williams, D. J.; Casson, J. L.; Schaller, R. D.; Klimov, V. I.; Hollingsworth, J. A. Utilizing the Lability of Lead Selenide to Produce Heterostructured Nanocrystals with Bright, Stable Infrared Emission. J. Am. Chem. Soc. 2008, 130, 4879-4885.

${ }^{4}$ Zhao, H.; Chaker, M.; Wu, N.; Ma, D. Towards Controlled Synthesis and Better Understanding of Highly Luminescent PbS/CdS Core/Shell Quantum Dots J. Mater. Chem. 2011, 21, 8898-8904.

${ }^{5}$ Friend, R. H.; Gymer, R. W.; Holmes, A. B.; Burroughes, J. H.; Marks, R. N.; Taliani, C.; Bradley, D. D. C.; Dos Santos, D. A.; Bredas, J. L.; Logdlund, M.; Salaneck, W. R. Electroluminescence in Conjugated Polymers. Nature 1999, 397, 121-128.

${ }^{6}$ Jasieniak, J.; Padicifico, J.; Signorini, R.; Chiasera, A.; Ferrari, M.; Martucci, A.; Mulvaney, P. Luminescence and Amplified Stimulated Emission in CdSe-ZnS-Nanocrystal-Doped $\mathrm{TiO}_{2}$ and $\mathrm{ZrO}_{2}$ Waveguides. Adv. Funct. Mater. 2007, 17, 1654.-1662

${ }^{7}$ Pang, L.; Shen, Y.; Tetz, K.; Fainman, Y. PMMA Quantum Dots Composites Fabricated via Use of Pre-polymerization. Opt. Express. 2005, 13, 44-49.

8 Suárez, I.; Gordillo, H.; Abargues, R.; Albert, S.; Martínez-Pastor, J. Photoluminescence Waveguiding in CdSe and CdTe QDs-PMMA Nanocomposite Films. Nanotechnology 2011, 22, 435202-8.

${ }^{9}$ Chang, T.-W. F.; Maria, A.; Cyr, P. W.; Sukhovatkin, V.; Levina, L.; Sargent, E. H. High nearinfrared photoluminescence quantum efficiency from $\mathrm{PbS}$ nanocrystals in polymer films. Synthetic Metals, 2005, 148, 257-261. 
10 Wang, M.; Zhang, M.; Qian, J.; Zhao, F.; Shen, L.; Scholes, G.; Winnik, M. Enhancing the Photoluminescence of Polymer-Stabilized $\mathrm{CdSe} / \mathrm{CdS} / \mathrm{ZnS}$ Core/Shell/Shell and CdSe/ZnS Core/Shell Quantum Dots in Water through a Chemical-Activation Approach. Langmuir 2009, 25, 11732-11740.

${ }^{11}$ Sundar, V. C.; Eisler, H. J.; Bawendi, M. G. Room-Temperature, Tunable Gain Media from Novel II-VI Nanocrystal-Titania Composite Matrices. Adv. Mater. 2002, 14, 739-743.

${ }^{12}$ Petruska, M. A.; Malko, A. V.; Voyles, P. M.; Klimov, V. I. High-Performance, Quantum Dot Nanocomposites for Nonlinear Optical and Optical Gain Applications. Adv. Mater, 2003, 15, 610613.

${ }^{13}$ Liao, Y.; Xu, Y.; Chan, Y. Semiconductor Nanocrystals in Sol-gel Derived Matrices. Phys.Chem.Chem.Phys, 2013, 15, 13694-13704.

${ }^{14}$ Tetsuka, H.; Ebina, T.; Mizukami, F. Highly Luminescent Flexible Quantum Dot-Clay Films. Adv. Mater. 2008, 20, 3039-3043.

15 Yoon, M.; Kim, Y.; Cho, J. Multifunctional Colloids with Optical, Magnetic, and Superhydrophobic Properties Derived from Nucleophilic Substitution-Induced Layer by-Layer Assembly in Organic Media. ACS Nano 2011, 5, 5417-5426.

${ }^{16}$ Liu, B.; Ren, T.; Zhang, J. R.; Chen, H. Y.; Zhu, J. J.; Burda, C. Spectroelectrochemistry of Hollow Spherical CdSe Quantum Dot Assemblies in Water. Electrochem. Commun. 2007, 9, 551557.

${ }^{17}$ Kovalenko, M. V.; Scheele, M.; Talapin, D. V. Colloidal Nanocrystals with Molecular Metal Chalcogenide Surface Ligands. Science 2009, 324, 1417-1420.

18 Talapin, D. V.; Lee, J. S.; Kovalenko, M. V.; Shevchenko, E. V. Prospects of Nanocrystal Solids as Electronic and Optoelectronic Materials. Chem. Rev. 2010, 110, 389-458.

${ }^{19}$ Humer, M.; Guider, R.; Jantsch, W.; Fromherz, T. Integration, Photostability and Spontaneous Emission Rate Enhancement of Colloidal PbS Nanocrystals for Si-based Photonics at Telecom Wavelengths. Opt. express 2013, 21, 18680-18688.

20 Novak, S.; Scarpantonio, L.; Novak, J.; Dai Pre, M.; Martucci, A.; Musgraves, J. D.; McClenaghan, N. D.; Richardson, K. Incorporation of Luminescent CdSe/ZnS Core-shell Quantum Dots and PbS Quantum Dots into Solution-derived Chalcogenide Glass Films. Opt. Mater. Express 2013, 3, 729-738. 
${ }^{21}$ Kovalenko, M. V. Chemical Design of Nanocrystal Solids. Chimia 2013, 67, 316-321.

${ }^{22}$ Steckel, J.S.; Coe-Sullivan, S.; Bulović, V.; Bawendi, M. Adv. Mater. 2003,15, 1862.

${ }^{23}$ Wehrenberg, B.L.; Wang, C.; Guyot-Sionnest, P. J. Phys. Chem. B, 2002, 106, 10634.

${ }^{24}$ Tsokkou, D.; Papagiorgis, P.; Protesescu, L.; Kovalenko, M.V.; Choulis, S.A.; Christofides, C.; Itskos, G.; Othonos. A. Photophysics of PbS Quantum Dot Films Capped with Arsenic Sulfide Ligands. Advanced Energy Materials. 2014, 1301547.

${ }^{25}$ Lambright, S.; Butaeva, E. V.; Razgoniaeva, N.; Hopkins, T.; Smith, B.; Perera, D. N.; Corbin, J.; Khon, E.; Thomas, R.; Moroz, P.; Mereshchenko, A. S.; Tarnovsky, A. N.; Zamkov, M. Enhanced Lifetime of Excitons in Non-Epitaxial Au/CdS Core/Shell Nanocrystals. ACS Nano 2014, $8,352-361$.

${ }^{26}$ Zhitomirsky, D.; Voznyy, O.; Hoogland, S.; Sargent, E. H. Measuring Charge Carrier Diffusion in Coupled Colloidal Quantum Dot Solids. ACS Nano, 2013, 7, 5282-5290.

${ }^{27}$ Chandler, R. E.; Houtepen, A. J.; Nelson, J.; Vanmaekelbergh, D. Electron Transport in Quantum Dot Solids: Monte Carlo Simulations of the Effects of Shell Filling, Coulomb Repulsions, and Site Disorder. Phys. Rev. B 2007, 75, 085325-10.

${ }^{28}$ Fafarman, A. T.; Koh, W. K.; Diroll, B. T.; Kim, D. K.; Ko, D. K.; Oh, S. J.; Ye, X. C.; DoanNguyen, V.; Crump, M. R.; Reifsnyder, D. C.; Murray, C. B.; Kagan, C. R. Thiocyanate-Capped Nanocrystal Colloids: Vibrational Reporter of Surface Chemistry and Solution-Based Route to Enhanced Coupling in Nanocrystal Solids. J. Am. Chem. Soc.2011, 133, 15753- 15761.

${ }^{29}$ Lee, J. S.; Kovalenko, M. V.; Huang, J.; Chung, D. S.; Talapin, D. V. Band-like Transport, High Electron Mobility and High Photoconductivity in All-Inorganic Nanocrystal Arrays. Nat. Nanotechnol.2011, 6, 348-352

${ }^{30}$ Liu, Y.; Tolentino, J.; Gibbs, M.; Ihly, R.; Perkins, C. L.; Liu, Y.; Crawford, N.; Hemminger,J. C.; Law, M. PbSe Quantum Dot Field-Effect Transistors with Air-Stable Electron Mobilities above 7 $\mathrm{cm}^{2} \mathrm{~V}^{-1} \mathrm{~s}^{-1}$. Nano Lett. 2013, 13, 1578- 1587

31 Tangirala, R.; Baker, J. L.; Alivisatos, A. P.; Milliron, D. J. Modular Inorganic Nanocomposites by Conversion of Nanocrystal Superlattices. Angew. Chem., Int. Ed. 2010, 49, 2878- 2882

32 Nag, A.; Chung, D. S.; Dolzhnikov, D. S.; Dimitrijevic, N. M.; Chattopadhyay, S.; Shibata, T.; Talapin, D. V. Effect of Metal Ions on Photoluminescence, Charge Transport, Magnetic and 
Catalytic Properties of All-Inorganic Colloidal Nanocrystals and Nanocrystal Solids. J. Am. Chem. Soc. 2012, 134, 13604-13615.

33 Llordes, A.; Garcia, G.; Gazquez, J.; Milliron, D. J. Tunable Near-Infrared and Visible-Light Transmittance in Nanocrystal-in-Glass Composites. Nature 2013, 500, 323-326.

${ }^{34}$ Khon, E.; Lambright, S.; Khon, D.; Smith, B.; O’Connor, T.; Moroz, P.; Imboden, M.; Diederich, G.; Perez-Bolivar, D.; Anzenbacher, P.; Zamkov, M. Inorganic Solids of CdSe Nanocrystals Exhibiting High Emission Quantum Yield. Adv. Funct. Mater. 2012, 22, 3714-3722.

${ }^{35}$ Diederich, G.; O’Connor, T.; Moroz, P.; Kinder, E.; Khon, E.; Perera, D.; Lorek, R.; Lambright, S.; Imboden, M.; Zamkov, M. Harvesting Solar Energy by Means of Charge-Separating Nanocrystals and Their Solids. J. Vis. Exp. 2012, 66, e4296.

${ }^{36}$ Pathan, H. M.; Lokhande, C. D. Deposition of Metal Chalcogenide Thin Films by Successive Ionic Layer Adsorption and Reaction (SILAR) Method. Bull. Mater. Sci. 2004, 27, 85-111.

37 Manna, L.; Scher, E. C.; Li, L.-S.; Alivisatos, A. P. Epitaxial Growth and Photochemical Annealing of Graded CdS/ZnS Shells on Colloidal CdSe Nanorods. J. Am. Chem. Soc. 2002, 124, $7136-7145$.

38 Carbone, L.; Cozolli, P. D. Colloidal Heterostructured Nanocrystals: Synthesis and Growth Mechanisms. Nano Today, 2010, 5, 449-493.

39 Donega, C. D. Synthesis and Properties of Colloidal Heteronanocrystals. Chem. Soc. Rev. 2011, 40, 1512- 1546.

${ }^{40}$ Moroz, P.; Kholmicheva, N.; Mellott, B.; Liyanage, G.; Rijal, U.; Bastola, E.; Huband, K.; Khon, E.; McBride, K.; Zamkov, M. Suppressed Carrier Scattering in CdS-Encapsulated PbS Nanocrystal Films. ACS Nano 2013, 7, 6964-6977.

${ }^{41}$ Helms, V. Fluorescence Resonance Energy Transfer". Principles of Computational Cell Biology. Weinheim: Wiley-VCH. p. 202. 2008

${ }^{42}$ Hines, M. A.; Scholes, G. D. Colloidal PbS Nanocrystals with Size-Tunable Near-Infrared Emission: Observation of Post-Synthesis Self-Narrowing of the Particle Size Distribution. Adv. Mater. 2003, 15, 1844-1849.

43 Yu, W. W.; Peng, X. Formation of High-Quality CdS and Other II - VI Semiconductor Nanocrystals in Noncoordinating Solvents: Tunable Reactivity of Monomers. Angew. Chem. 2002, 114, 2474-2477. 
${ }^{44}$ Kinder, E.; Moroz, P.; Diederich, G.; Johnson, A.; Kirsanova, M.; Nemchinov, A.; O’Connor, T.; Roth, D.; Zamkov, M. Fabrication of All-Inorganic Nanocrystal Solids through Matrix Encapsulation of Nanocrystal Arrays. J. Am. Chem. Soc. 2011, 133, 20488-20499

${ }^{45}$ Semonin, O. E.; Johnson, J. C.; Luther, J. M.; Midgett, A. G.; Nozik, A. J.; Beard, M. C. Absolute Photoluminescence Quantum Yields of IR-26 Dye, PbS, and PbSe Quantum Dots J. Phys. Chem. Lett. 2010, 1, 2445-2450. 